\title{
Efficacy and safety of erythropoietin and iron therapy to reduce red blood cell transfusion in surgical patients: a systematic review and meta-analysis \\ Efficacité et innocuité d'un traitement d'érythropoïétine et de fer pour réduire la transfusion de culots sanguins chez les patients chirurgicaux: une revue systématique et méta-analyse
}

\author{
Tiffanie Kei, MHSc • Nikhil Mistry, MSc • Gerard Curley, MB, MSc, PhD • Katerina Pavenski, MD • \\ Nadine Shehata, MD • Rosa Maria Tanzini, BSc (Pharm) • Marie-France Gauthier, PharmD, ACPR • \\ Kevin Thorpe, MMath • Tom A. Schweizer, PhD • Sarah Ward, MD • C. David Mazer, MD • \\ Gregory M. T. Hare, MD, PhD
}

Received: 24 July 2018/Revised: 19 December 2018/Accepted: 29 December 2018/Published online: 28 March 2019

(C) Canadian Anesthesiologists' Society 2019

\begin{abstract}
Purpose Iron restricted anemia is prevalent in surgical patients and is associated with an increased risk of allogeneic red blood cell $(R B C)$ transfusion and adverse
\end{abstract}

Preliminary data from this research were presented by Tiffanie Kei in the Richard Knill Competition at the Canadian Anaesthesiologists' meeting, Niagara Falls 2017.

Electronic supplementary material The online version of this article (https://doi.org/10.1007/s12630-019-01351-6) contains supplementary material, which is available to authorized users.

Tiffanie Kei and Nikhil Mistry contributed equally to the data assessment, analysis, and formulation of this manuscript.

T. Kei, MHSc $\cdot$ N. Mistry, MSc

Department of Anesthesia, St. Michael's Hospital, University of

Toronto, Toronto, ON, Canada

G. Curley, MB, MSc, PhD

Department of Anesthesia and Critical Care Medicine, Royal College of Surgeons in Ireland, Dublin, Ireland

\section{K. Pavenski, MD}

Department of Laboratory Medicine and Pathobiology, St.

Michael's Hospital, University of Toronto, Toronto, ON, Canada

St. Michael's Hospital, Centre of Excellence in Patient Blood

Management, Toronto, ON, Canada

N. Shehata, MD

Departments of Medicine and Laboratory Medicine and Pathobiology, Institute of Health Policy Management and

Evaluation, Mount Sinai Hospital, Toronto, ON, Canada events. Treatment of anemia includes oral and intravenous iron and erythropoiesis stimulating agents (ESAs). More recent studies have focused on the use of intravenous iron as the primary approach to treating anemia. Nevertheless, the optimal treatment strategy for anemia remains to be established. Our primary objective was to evaluate the efficacy and safety of ESA and iron therapy relative to iron therapy alone in reducing $R B C$ transfusion in surgical patients.

Source We searched the Cochrane Library, MEDLINE, EMBASE, and ClinicalTrials.gov from inception to May 2018. We included randomized-controlled trials in which adult surgical patients received an ESA and iron, vs iron

\section{R. M. Tanzini, BSc (Pharm)}

Department of Pharmacy, St. Michael's Hospital, Toronto, ON, Canada

M.-F. Gauthier, PharmD, ACPR

Department of Pharmacy, Montfort Hospital, Ottawa, ON, Canada

K. Thorpe, MMath

Applied Health Research Centre, St. Michael's Hospital, Dalla Lana School of Public Health, University of Toronto, Toronto, ON, Canada

T. A. Schweizer, $\mathrm{PhD}$

Keenan Research Centre for Biomedical Science in the Li Ka Shing Knowledge Institute of St. Michael's Hospital, Toronto, ON, Canada 
alone, prior to cardiac and non-cardiac surgery. Our primary outcome was RBC transfusion rate. Secondary outcomes included hemoglobin concentration (posttreatment and postoperatively), number of RBC units transfused, mortality, stroke, myocardial infarction (MI), renal dysfunction, pulmonary embolism (PE), and deep vein thrombosis (DVT).

Principal findings In total, 25 studies (4,719 participants) were included. Erythropoiesis stimulating agents and iron therapy reduced $R B C$ transfusion relative to iron therapy (relative risk [RR] 0.57; 95\% confidence interval [CI], 0.46 to 0.71$)$ without any change in mortality (RR 1.31; 95\% CI, 0.80 to 2.16), stroke (RR 1.91; 95\% CI, 0.63 to 5.76), MI (RR 1.12; 95\% CI, 0.50 to 2.50), renal dysfunction (RR 0.96; 95\% CI, 0.72 to 1.26), PE (RR 0.92; $95 \%$ CI, 0.15 to 5.83), or DVT (RR 1.48; 95\% CI, 0.95 to 2.31 ).

Conclusion Administration of ESA and iron therapy reduced the risk for RBC transfusion compared with iron therapy alone in patients undergoing cardiac and noncardiac surgery. Nevertheless, publication bias and heterogeneity reduces the confidence of the finding. Although the analysis was probably under-powered for some outcomes, no difference in the incidence of serious adverse events was observed with ESA and iron compared with iron alone. Further large prospective trials are required to confirm these findings.

\section{Résumé}

Objectif L'anémie ferriprive est prévalente chez les patients chirurgicaux et est associée à un risque accru de transfusion de culots sanguins allogènes et d'événements indésirables. Le traitement de l'anémie comprend la prise orale et intraveineuse de fer et d'agents stimulant l'érythropoïèse (ASE). Les études plus récentes se sont concentrées sur l'utilisation de fer intraveineux en tant que thérapie principale pour traiter l'anémie. Toutefois, la stratégie thérapeutique optimale pour l'anémie demeure inconnue. Notre objectif principal était d'évaluer

\section{S. Ward, MD}

Division of Orthopedic surgery, Department of Surgery, St. Michael's Hospital, University of Toronto, Toronto, ON, Canada

C. D. Mazer, MD

Department of Anesthesia, St. Michael's Hospital, University of Toronto, Toronto, ON, Canada

Keenan Research Centre for Biomedical Science in the Li Ka Shing Knowledge Institute of St. Michael's Hospital, Toronto, ON, Canada

Department of Physiology, University of Toronto, Toronto, ON, Canada l'efficacité et l'innocuité des ASE et du traitement de fer par rapport à un traitement à base de fer seulement afin de réduire les transfusions de culots sanguins chez les patients chirurgicaux.

Source Nous avons effectué des recherches dans les bases de données suivantes: Cochrane Library, MEDLINE, EMBASE et ClinicalTrials.gov, de leur création au mois de mai 2018. Nous avons inclus les études randomisées contrôlées dans lesquelles des patients chirurgicaux adultes ont reçu des ASE et du fer vs du fer seulement, avant une chirurgie cardiaque ou non cardiaque. Notre critère d'évaluation principal était le taux de transfusion de culots sanguins. Nos critères secondaires comprenaient la concentration d'hémoglobine (post-traitement et en postopératoire), le nombre d'unités de culots sanguins transfusées, la mortalité, les accidents vasculaires cérébraux (AVC), les infarctus du myocarde (IM), le dysfonctionnement rénal, l'embolie pulmonaire (EP) et la thrombose veineuse profonde (TVP).

Constatations principales $A u$ total, 25 études $(4719$ participants) ont été retenues. Le traitement à base d'agents stimulant l'érythropoïèse et de fer a réduit les transfusions de culots sanguins par rapport au traitement à base de fer seul (risque relatif [RR] 0,57; intervalle de confiance [IC] $95 \%, 0,46$ à 0,71), sans changement au niveau de la mortalité (RR 1,31; IC $95 \%, 0,80$ à 2,16), des AVC (RR 1,91; IC $95 \%, 0,63$ à 5,76), des IM (RR 1,12; IC $95 \%, 0,50$ à 2,50), du dysfonctionnement rénal (RR 0,96; IC $95 \%, 0,72$ à 1,26), de l'EP (RR 0,92; IC $95 \%, 0,15$ à 5,83), ou de la TVP (RR 1,48; IC $95 \%, 0,95$ à 2,31).

Conclusion L'administration d'un traitement d'ASE et de fer a réduit le risque de transfusion de culots sanguins par rapport à un traitement à base de fer uniquement chez les patients subissant une chirurgie cardiaque ou non cardiaque. Toutefois, le biais de publication et l'hétérogénéité des études réduisent la fiabilité de cette conclusion. Bien que notre analyse manquait probablement de puissance quant à certains critères d'évaluation, aucune différence dans l'incidence d'événements indésirables

G. M. T. Hare, MD, PhD ( $ه)$

Department of Anesthesia, St. Michael's Hospital, University of Toronto, Toronto, ON, Canada

e-mail: hareg@smh.ca

Keenan Research Centre for Biomedical Science in the Li Ka Shing Knowledge Institute of St. Michael's Hospital, Toronto, ON, Canada

St. Michael's Hospital, Centre of Excellence in Patient Blood Management, Toronto, ON, Canada

Department of Physiology, University of Toronto, Toronto, ON, Canada 
graves n'a été observée avec l'administration d'ASE et de fer par rapport à une administration de fer seul. D'autres études prospectives d'envergure sont nécessaires afin de confirmer ces résultats.

Preoperative anemia has been associated with increased morbidity and mortality for patients undergoing cardiac ${ }^{1,2}$ and non-cardiac ${ }^{3-8}$ surgery. The prevalence of preoperative anemia is estimated to be near $30 \%$ in these patient populations. ${ }^{2,8-10}$ The majority of these anemic patients have a diagnosis of iron restricted anemia, which includes those with absolute iron deficiency anemia (IDA) and anemia of chronic inflammation (ACI). ${ }^{11}$ Effective treatments for these anemias include iron and erythropoiesis stimulating agents $(\mathrm{ESA}){ }^{12,13}$ Expert opinion and consensus guidelines advocate for oral or intravenous iron alone as first line therapies for IDA, and limit the recommended use of ESA for refractory patients with a diagnosis of ACI. ${ }^{14}$

The efficacy of iron therapy alone has been assessed in previously published meta-analyses. ${ }^{15-18}$ These analyses showed a trend toward increased postoperative hemoglobin concentration $(\mathrm{Hb})$ but did not show a clear effect on red blood cell (RBC) transfusion avoidance. ${ }^{16-18}$ These clinical studies and a recently published small randomizedcontrolled trial (RCT) also support the finding that intravenous iron may be more effective than oral iron at restoring iron homeostasis. ${ }^{17,19}$

Previous systematic reviews and meta-analyses have directly assessed the efficacy of ESAs with respect to RBC avoidance. ${ }^{20-22}$ Nevertheless, these studies were limited in that: 1) all relevant RCTs that assessed the efficacy of preoperative ESA therapy on RBC transfusion avoidance were not included ${ }^{20-22}$; 2) they included studies that utilized preoperative autologous donation (PAD), which may have confounded the impact of ESAs on RBC utilization and $\mathrm{Hb}$ outcomes ${ }^{20,21}$; 3) one study did not perform a formal meta-analysis ${ }^{22}$; 4) data on all cardiac and non-cardiac surgical patient populations were not included; and 5) complete data on serious adverse effects associated with ESA therapy were not included ${ }^{20-22}$ (available as Electronic Supplementary Material [ESM] eAppendix, eTable 2 and eTable 3).

Currently, the use of ESA to manage preoperative anemia has been tempered because of: 1) the impression that intravenous iron therapy alone may be adequate as an effective mono-therapy for anemia (PREVENTT, NCT01692418; ITACS, NCT02632760) $)^{23}$; and 2) concerns about the potential for increased rates of thrombosis, stroke, and mortality with the use of high dose ESA therapy in medical patients. ${ }^{24-26}$ These complications may not apply to patients treated with ESA therapy for preoperative anemia given that: 1) the doses used are relatively low; 2) the duration of therapy is typically short, and 3) there are acute hemodynamic responses to surgical blood loss and hemodilution (increased cardiac output and tissue blood flow), which would reduce the potential risk of thrombosis.

To address this lingering uncertainty, we conducted the current systematic review and meta-analysis to assess the relative efficacy and safety of ESA and iron $v s$ iron alone in perioperative patients undergoing cardiac and non-cardiac surgery. We hypothesized that ESA and iron treatment combined would be more efficacious relative to iron therapy alone in terms of avoiding RBC transfusion.

\section{Methods}

The predefined review protocol was registered at the PROSPERO international prospective register of systematic reviews (http://www.crd.york.ac.uk/ PROSPERO), registration number CRD42015025870. The initial study design for this manuscript included a comparison of the use of ESA and iron with iron alone and the use of iron $v s$ placebo. Based on the reviewers' comments in the initial peer review of our manuscript and an analysis on existing systematic reviews and meta-analyses of published trials comparing outcomes associated with iron $v s$ placebo, we modified the primary objective in the revised version of our manuscript to evaluate the efficacy and safety of the preoperative use of ESA and iron combined, relative to iron alone, focusing on the incidence of any $R B C$ transfusion as the primary outcome for efficacy. Secondary outcomes included post-treatment changes in $\mathrm{Hb}$ level, postoperative $\mathrm{Hb}$ concentration, number of $\mathrm{RBC}$ units transfused, mortality, stroke, MI, renal dysfunction, pulmonary embolism (PE), and deep vein thrombosis (DVT).

\section{Search strategy}

We searched three databases for relevant studies, MEDLINE (Ovid) from 1946 until May 2018, EMBASE from 1947 until May 2018, and Cochrane databases (Database of Systematic Reviews, Central Register of Controlled Trials) until May 2018 (eAppendix, available as ESM). We also searched for ongoing or completed trials on ClinicalTrials.gov. The search strategy was adapted for each database using groups of keywords for iron, erythropoietin (EPO), anemia, and surgery. We did not restrict our search for trials by date, language, or publication status. We also searched the reference lists of 
relevant reviews, published papers, as well as the reference lists of all included trials for further studies. The full search strategy is available in the ESM.

\section{Eligibility criteria}

To be included in the systematic review, the following criteria had to be met: 1) study design: RCT; 2) patient population: adult patients undergoing surgical interventions including, but not limited to, cardiac, orthopedic, and colorectal cancer operations; 3) intervention: perioperative treatment with ESA and iron combined relative to iron alone; 4) outcomes: reporting of at least one of the primary or secondary outcomes. We expected the definitions of each of the morbidity events to vary between studies. Studies were excluded if: 1) the effect of iron and/or ESA could not be elicited because of to multiple interventions; 2) we were unable to distinguish the patients of interest from the larger study population; 3 ) patients underwent acute normovolemic hemodilution; 4) patients received ESA and iron therapy only during the intraoperative or postoperative periods; or 5) patients were enrolled in PAD programs, which would confound the ability to assess the effect of ESA and iron therapy on RBC transfusion, RBC utilization, post-treatment $\mathrm{Hb}$ concentration, and postoperative $\mathrm{Hb}$ concentration. In the event of duplicate publication, we included the study with the most complete data.

\section{Primary and secondary outcomes}

The primary outcome for this study was the proportion of patients receiving perioperative allogeneic $\mathrm{RBC}$ transfusion. Secondary outcomes included post-treatment $\mathrm{Hb}$ concentration (change in $\mathrm{Hb}$ concentration from baseline to time of surgery), number of RBC units transfused, postoperative $\mathrm{Hb}$ concentration, mortality, stroke, myocardial infarction (MI), renal dysfunction, DVT, and PE. The relative change in reticulocyte count was also included, but not meta-analyzed because of inconsistency in the format of outcome reporting.

\section{Study selection and data extraction}

Three reviewers (T.K., N.M., and G.M.T.H.) independently screened citations to select trials that met inclusion criteria and abstracted data using a structured data extraction form. Disagreements on study inclusion or endpoints were resolved by a fourth author (G.C.). The three reviewers independently extracted study characteristics and outcomes including study design, methodology, type of surgery, patient characteristics, and outcomes.

We developed a standardized extraction form following the recommendations of the Cochrane Effective Practice and Organization of Care Review Group. We determined study design using the Cochrane Group checklist. ${ }^{27}$ The following patient characteristics were captured: indication for and site of surgery, age, and sex. We collected intervention and comparator information, including type of iron and ESA, route of administration, dosage, dosing interval, timing of administration, type of comparator (oral/ intravenous iron, placebo, or standard care), and use of cointerventions.

\section{Quality and risk of bias assessment}

The methodologic quality of individual studies was assessed using the Cochrane Collaboration's tool for assessing risk of bias as described in section 8.5 of the Cochrane Handbook for Systematic Reviews of Interventions. ${ }^{28} \mathrm{We}$ assessed the following domains for each study: sequence generation; allocation concealment; blinding; incomplete outcome data; selective outcome reporting; other potential sources of bias (e.g., potential conflict(s) of interest). We completed a "Risk of bias" table for each study, incorporating a description of the study's performance against each of the above domains and our overall judgment of the risk of bias for each entry as follows: "Low", "Unclear", and "High" risk of bias. ${ }^{28} \mathrm{~A}$ study was considered to have an overall low risk of bias if at least six of the seven domains were low risk. A domain was considered to be overall low risk if $>50 \%$ of the studies were assessed as low risk.

\section{Data synthesis}

We combined data from all studies to estimate the pooled relative risk (RR) and associated $95 \%$ confidence intervals (CI) for the binary outcomes of patients transfused, mortality, MI, renal dysfunction, stroke, DVT, and PE. We used the pooled weighted mean difference with $95 \%$ CIs to estimate the effect on the continuous outcomes of RBC units transfused and $\mathrm{Hb}$ levels. Pooled RRs and mean differences were estimated by the inverse variance approach using the random-effects model of DerSimonian and Laird ${ }^{29}$ to estimate variances. When only one group had no events, then 0.5 was added to each cell to allow estimation of the RR. Heterogeneity was tested by a weighted inverse variance Chi-squared test and quantified by $\mathrm{I}^{2}$, which is the percentage of total between-study variability due to heterogeneity rather than chance. ${ }^{30}$ Substantial heterogeneity is considered to exist when the $\mathrm{I}^{2}>50 \%$. For the Chi-squared test, we used a $P$ value of $<$ 0.10 to indicate the presence of statistically significant heterogeneity. All analyses were conducted using Review Manager 5.3 software (the Cochrane Collaboration, UK). We considered $P<0.05$ to be statistically significant. Data 
presented only in figures were extracted to numerical values, and missing standard deviations were imputed wherever possible. Medians, interquartile ranges, standard errors, and $95 \%$ CIs were converted to means and standard deviations using previously published methods ${ }^{31}$ and as outlined in the Cochrane Handbook (sections 7.7.3.2, 7.7.3.5, and 16.1.3.2). In studies assessing the effect of multiple doses of ESA vs placebo, the ESA subgroups were combined using the formulae outlined in Table 7.7.a of the Cochrane Handbook. Publication bias was assessed with a funnel plot, plotting the RR for proportion transfused against the standard error of the log RR.

\section{Subgroup and sensitivity analysis}

We defined a priori subgroup analyses across the two interventions: ESA and oral iron $v s$ oral iron alone, and ESA and intravenous iron $v s$ intravenous iron alone. Furthermore, a post-hoc subgroup analysis across orthopedic, cardiac, and colorectal cancer surgical subtypes was also performed. Anticipating a significant degree of heterogeneity in our primary outcomes, sensitivity analyses were performed: 1) by dose of ESA (low dose [total ESA dose $\leq 80,000$ IU] $v s$ high dose [total ESA dose $>80,000 \mathrm{IU}]$ ); and 2) by excluding studies with a high risk of bias from the primary analysis. Interpretation of subgroup analysis was guided by recently published recommendations. ${ }^{32}$

\section{Results}

Search results

A total of 3,449 articles were retrieved during the initial search. After removing duplicates, 3,297 articles were screened based on titles and abstracts, and 88 were assessed in full-text. Following full-text screening, 63 articles were excluded, 16 of which included patients that did not undergo the interventions of interest, seven of which administered ESA and iron therapy only intraoperatively or postoperatively, 39 of which enrolled patients in PAD programs, and one which reported the long-term outcomes of an excluded study. A total of 25 studies (4,719 patients) underwent full-text screening and were included in this meta-analysis (Fig. 1). ${ }^{33-57}$

\section{Characteristics of included trials}

All studies were divided into two main subgroups: ESA with oral iron $v s$ oral iron alone, and ESA with intravenous iron $v s$ intravenous iron alone. The characteristics of the included trials are summarized in Tables 1 and 2. The surgical populations were primarily cardiac (six RCTs), ${ }^{34,43,45,46,51,57 \quad \text { orthopedic (ten }}$ RCTs), ${ }^{33,35,36,44,48-50,52,53,55}$ and colorectal cancer (four RCTs). ${ }^{37,42,47,56}$ One trial reported results from a surgical population of both cardiac and orthopedic patients. ${ }^{40}$ Four trials reported outcomes from other surgical procedures. ${ }^{38,39,41,54}$ Eighteen RCTs assessed ESA with oral iron $v s$ oral iron therapy (Table 1). ${ }^{33-50}$ Seven RCTs assessed ESA and intravenous iron vs intravenous iron therapy (Table 2). ${ }^{51-57}$ One trial compared ESA with oral and intravenous iron $v s$ oral iron therapy. ${ }^{57}$ One trial assessed both ESA and oral iron and ESA and intravenous iron by randomizing patients to one of four interventions: 1) ESA and oral iron; 2) oral iron; 3) ESA and intravenous iron; and 4) intravenous iron. ${ }^{55}$ For this study, the ESA and oral iron subgroup was analyzed separately from the ESA and intravenous iron subgroup for the following analyses: change in post-treatment $\mathrm{Hb}$ concentration, $\mathrm{RBC}$ units transfused, postoperative $\mathrm{Hb}$ concentration, mortality, stroke, MI, renal dysfunction, PE, and DVT.

Quality assessment and risk of bias

The risk of bias of the included studies is described in the ESM (eFig. 1 and eFig. 2). The risk of bias was graded as being low for most categories with the exception of allocation concealment and blinding of outcome assessment, which were either unclear, not explicitly stated, or at high risk. Most studies explicitly described their method of random allocation and blinding of participants and personnel, although blinding of outcome assessment was largely unclear or missing from over $75 \%$ of studies.

\section{Quantitative analysis}

Efficacy of ESA and iron therapy: primary outcome-RBC transfusion

Erythropoiesis stimulating agents and iron therapy combined reduced the risk for RBC transfusion relative to iron therapy alone (RR, $0.57 ; 95 \% \mathrm{CI}, 0.46$ to $0.71, \mathrm{I}^{2}=$ $78 \%$; overall effect, $P<0.001,22$ studies reported this outcome) (Fig. 2). The test for subgroup differences between oral and intravenous iron did not reveal significant differences $(P=0.06)$ (Fig. 2). A reduction in RBC transfusion was observed when studies were divided by low EPO dose ( $\leq 80,000 \mathrm{IU}$, ten studies) vs high ESA dose ( $>$ 80,000 IU, 13 studies). The test for subgroup differences between low and high dose ESA was negative $(P=0.59)$ (Fig. 3). In a subgroup analysis, ESA plus iron therapy resulted in RBC transfusion avoidance in patients undergoing orthopedic and cardiac surgery, but not those 
Fig. 1 Flow diagram indicates the number of studies identified, screened, assessed for eligibility, and included in the meta-analysis

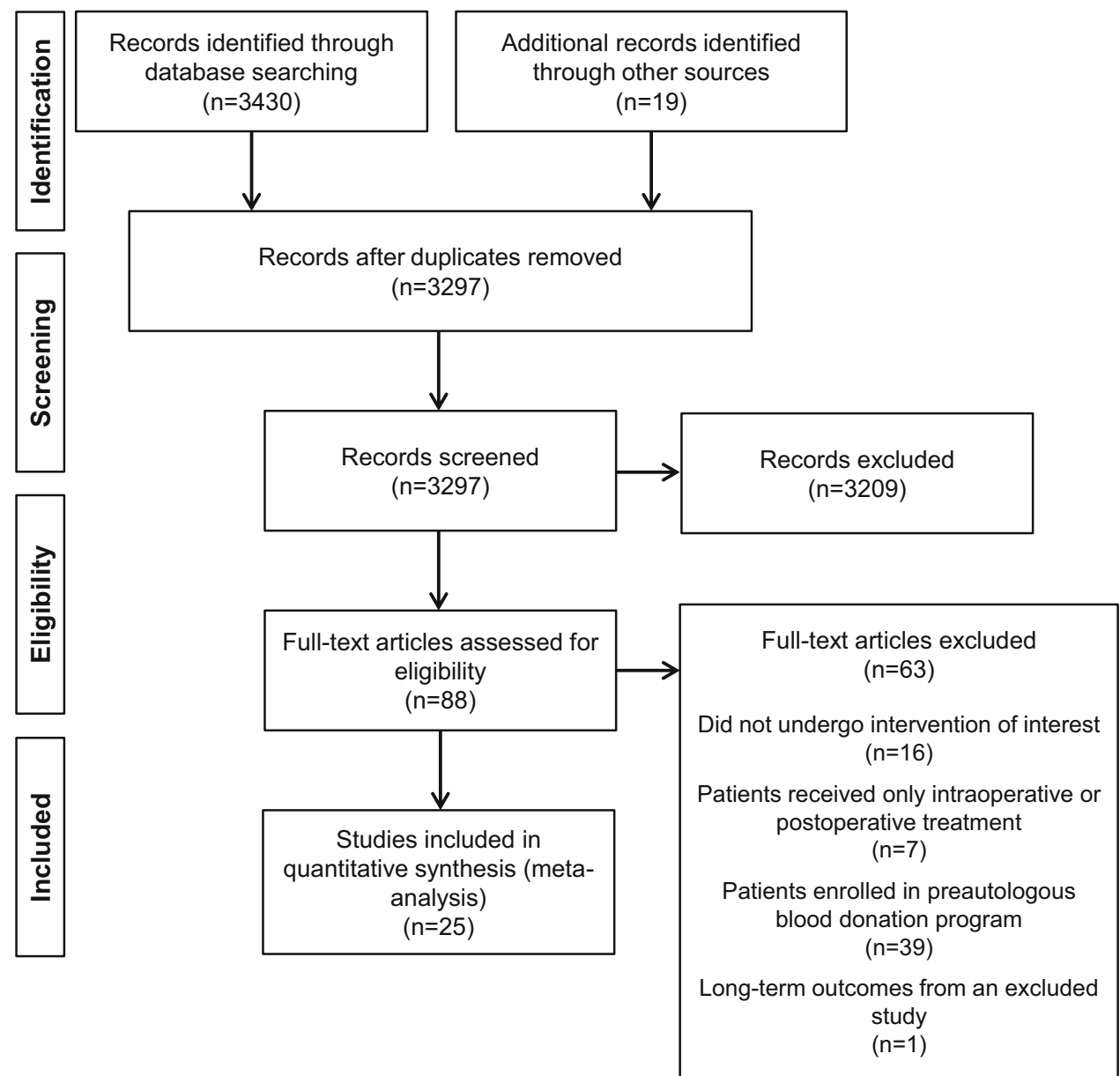

undergoing colorectal surgery. The test for subgroup differences between orthopedic, cardiac and colorectal surgery was positive $(P=0.03)$ (Fig. 4). There was a substantial amount of heterogeneity within each group of surgical interventions (orthopedic surgery $\mathrm{I}^{2}=89 \%$; cardiac surgery $\mathrm{I}^{2}=74 \%$; colorectal cancer surgery $\mathrm{I}^{2}=$ $82 \%$ ). Thus, the validity of the treatment effect estimate for each subgroup is uncertain. ${ }^{32}$

This effect on the primary outcome (RBC transfusion) was supported by a number of secondary outcomes including: 1) post-treatment $\mathrm{Hb}$ effect (mean difference in $\mathrm{Hb}$ concentration, $8.28 \mathrm{~g} \cdot \mathrm{L}^{-1}$; $95 \% \mathrm{CI}, 4.54$ to $12.02, \mathrm{I}^{2}=$ 98\%; overall effect, $P<0.001,22$ studies reported this outcome); 2) RBC units transfused (mean difference in RBC units transfused, -0.51 units; $95 \% \mathrm{CI},-0.70$ to -0.33 , $\mathrm{I}^{2}=71 \%$; overall effect, $P<0.001,18$ studies reported this outcome); and 3) postoperative $\mathrm{Hb}$ concentration (mean difference in $\mathrm{Hb}$ concentration, 9.01; 95\% CI, 6.58 to $11.44, \mathrm{I}^{2}=89 \%$; overall effect, $P<0.001,18$ studies reported this outcome) (ESM eFigs 3, 4, and 5, respectively). For all three of these secondary comparisons, there was no difference between oral vs intravenous iron treatment subgroups (subgroup difference
$P$ values; post-treatment $\mathrm{Hb}$ concentration, $P=0.18$; RBC units transfused, $P=0.86$ and postoperative $\mathrm{Hb}, P=0.12$ ). A crude, un-weighted assessment of the efficacy of ESA and iron $v s$ iron alone was obtained by comparing the relative reticulocyte response to therapy that was $170 \%$ vs $1 \%$ (ESM eTable 1).

\section{Adverse effects of ESA and iron therapy: secondary outcomes}

Erythropoiesis stimulating agents and iron therapy combined did not increase the risk for: 1) mortality (RR, $1.31 ; 95 \% \mathrm{CI}, 0.80$ to $2.16, \mathrm{I}^{2}=0 \%$; overall effect, $P=$ 0.29, 20 studies) (ESM eFig. 6); 2) stroke (RR, 1.91; 95\% CI, 0.63 to $5.76, \mathrm{I}^{2}=0 \%$; overall effect, $P=0.25,8$ studies) (ESM eFig. 7); 3) MI (RR, 1.12; 95\% CI, 0.50 to $2.50, \mathrm{I}^{2}=$ $0 \%$; overall effect, $P=0.79$, seven studies) (ESM eFig. 8); 4) renal dysfunction (RR, $0.96 ; 95 \% \mathrm{CI}, 0.72$ to $1.26, \mathrm{I}^{2}=$ $0 \%$; overall effect, $P=0.75$, four studies) (ESM eFig. 9); 5) PE (RR, 0.92; 95\% CI, 0.15 to $5.83, \mathrm{I}^{2}=0 \%$; overall effect, $P=0.93$, six studies) (ESM eFig. 10); and 6) DVT (RR, 1.48; $95 \% \mathrm{CI}, 0.95$ to $2.31, \mathrm{I}^{2}=0 \%$; overall effect, $P=$ $0.09,12$ studies) (ESM eFig. 11).When assessed by dose of 
Table 1 Characteristics of all randomized-controlled trials evaluating EPO and oral iron $v s$ iron studies

\begin{tabular}{|c|c|c|c|c|}
\hline Reference & Size & Population & $\begin{array}{l}\text { Intervention and } \\
\text { comparator }\end{array}$ & Outcomes \\
\hline Wu 2016 & $n=$ & $\begin{array}{l}\text { Male: } \mathrm{Hb} 150-130 \mathrm{~g} \cdot \mathrm{L}^{-1} \\
\text { Female: } \mathrm{Hb} 150- \\
\quad 120 \mathrm{~g} \cdot \mathrm{L}^{-1} \\
\text { Primary bilateral total } \\
\quad \text { hip arthroplasty }\end{array}$ & $\begin{array}{l}\text { EPO }(70,000 \mathrm{IU} ; n= \\
30) \\
\text { Oral iron }(n=32) \\
\text { Preoperative and } \\
\text { postoperative }\end{array}$ & $\begin{array}{l}\text { Hb concentration, reticulocyte count, Hct, RBC transfusion rate, } \\
\text { symptoms of anemia (dizziness and fatigue), DVT, pulmonary } \\
\text { embolism, blood loss, drainage volume }\end{array}$ \\
\hline Weltert 2015 & $\begin{array}{l}n= \\
600\end{array}$ & $\begin{array}{l}\mathrm{Hb}<145 \mathrm{~g} \cdot \mathrm{L}^{-1} \\
\text { Cardiac surgery }\end{array}$ & $\begin{array}{l}\text { EPO }(80,000 \mathrm{IU})+\text { oral } \\
\quad \text { iron }(n=300) \\
\text { Oral iron }(n=300) \\
\text { Preoperative only }\end{array}$ & $\begin{array}{l}\text { RBC transfusions, mortality and adverse events, } \mathrm{Hb} \text { on } \\
\text { postoperative day } 4 \text {, number of units of RBC transfused }\end{array}$ \\
\hline Stowell 2009 & $\begin{array}{l}n= \\
680\end{array}$ & $\begin{array}{l}\mathrm{Hb} 100-130 \mathrm{~g} \cdot \mathrm{L}^{-1} \\
\text { Elective spinal surgery }\end{array}$ & $\begin{array}{l}\text { EPO }(\sim 168,000 \mathrm{IU})+ \\
\quad \text { oral iron }(n=340) \\
\text { Oral iron }(n=340) \\
\text { Preoperative only }\end{array}$ & DVT and other thrombovascular events \\
\hline $\begin{array}{l}\text { Christodoulakis } \\
\quad 2005\end{array}$ & $n=$ & $\begin{array}{l}\mathrm{Hb} 90-120 \mathrm{~g} \cdot \mathrm{L}^{-1} \\
\text { Elective colorectal } \\
\text { cancer surgery }\end{array}$ & $\begin{array}{l}\text { EPO }(\sim 126,000 \mathrm{IU})+ \\
\quad \text { oral iron }(n=69) \\
\text { EPO }(\sim 252,000 \mathrm{IU})+ \\
\quad \text { oral iron }(n=67) \\
\text { Oral iron }(n=68) \\
\text { Preoperative and } \\
\quad \text { postoperative }\end{array}$ & $\begin{array}{l}\text { Transfusions, Hct, } \mathrm{Hb} \text {, reticulocyte count, mortality, } \\
\text { cardiorespiratory failure, atrial fibrillation, neutropenia, } \\
\text { peritonitis, urticarial, cardiac arrest, embolism }\end{array}$ \\
\hline Weber 2005 & $\begin{array}{l}n= \\
695\end{array}$ & $\begin{array}{l}\mathrm{Hb} 100-130 \mathrm{~g} \cdot \mathrm{L}^{-1} \\
\text { Elective major } \\
\text { orthopedic surgery } \\
\text { (hip, knee, spine) }\end{array}$ & $\begin{array}{l}\text { EPO }(160,000 \mathrm{IU})+ \\
\quad \text { oral iron }(n=460) \\
\text { Oral OR IV iron: }(n= \\
235) \\
\text { Preoperative only }\end{array}$ & $\begin{array}{l}\text { Transfusions, } \mathrm{Hb} \text {, time to ambulation and discharge, infections } \\
\text { (wound, abscess, infected hematoma, respiratory tract, urinary } \\
\text { tract, pneumonia, bacteremia), therapeutic antibiotic use, } \\
\text { thrombotic events }\end{array}$ \\
\hline Dousias 2003 & $n=$ & $\begin{array}{l}\text { Females, Hb } 90-120 \\
\text { g. } \mathrm{L}^{-1} \\
\text { Total hysterectomy } \\
\quad \text { (uterine leiomyoma) }\end{array}$ & $\begin{array}{l}\text { EPO }(\sim 126,000 \mathrm{U})+ \\
\quad \text { oral iron }(n=23) \\
\text { Oral iron }(n=27) \\
\text { Preoperative only }\end{array}$ & $\begin{array}{l}\text { Transfusions, Hb, reticulocyte, ferritin levels, length of } \\
\text { hospitalization }\end{array}$ \\
\hline Scott 2002 & $n=$ & $\begin{array}{l}\mathrm{Hb} 100-135 \mathrm{~g} \cdot \mathrm{L}^{-1} \\
\text { Major head and neck } \\
\text { oncologic surgery }\end{array}$ & $\begin{array}{l}\text { EPO }(\sim 126,000 \mathrm{IU})+ \\
\text { oral iron }(n=29) \\
\text { Placebo }+ \text { oral iron }(n= \\
29) \\
\text { Preoperative only }\end{array}$ & $\begin{array}{l}\text { Hb, Hct, reticulocytes, transfusions, adverse events (mortality, } \\
\text { thrombotic/vascular events [DVT], prolonged hypertension, } \\
\text { etc.) }\end{array}$ \\
\hline Larson 2001 & $n=$ & $\begin{array}{l}\text { Females, } \mathrm{Hb}<120 \\
\mathrm{~g} \cdot \mathrm{L}^{-1} \\
\text { Uterine myoma } \\
\text { scheduled for } \\
\text { hysterectomy }\end{array}$ & $\begin{array}{l}\text { EPO }(40,000 \mathrm{IU})+\text { oral } \\
\quad \text { iron; } n=15) \\
\text { Oral iron }(n=16) \\
\text { Preoperative only }\end{array}$ & $\begin{array}{l}\text { Transfusions, infections, Hb, Hct, blood pressure, platelets, white } \\
\text { blood cells, serum iron, ferritin, iron saturation, blood loss }\end{array}$ \\
\hline Wurnig 2001 & $n=$ & $\begin{array}{l}\text { Hct } 30-40 \% \\
\text { Any elective surgery } \\
\text { (mainly orthopedic or } \\
\text { cardiac) }\end{array}$ & $\begin{array}{l}\text { EPO }(\sim 30,625)+\text { oral } \\
\quad \text { iron }(n=70) \\
\text { EPO }(\sim 61,250)+\text { oral } \\
\quad \text { iron }(n=64) \\
\text { Oral iron }(n=60) \\
\text { Preoperative only }\end{array}$ & $\begin{array}{l}\text { Transfusions, hematologic parameters }(\mathrm{Hb}, \mathrm{Hct}) \text {, iron kinetics } \\
\text { (serum iron, serum ferritin, transferrin saturation), adverse } \\
\text { events (mortality, thrombotic/vascular events, hypertension, } \\
\text { etc.) }\end{array}$ \\
\hline Feagan 2000 & $n=$ & $\begin{array}{l}\mathrm{Hb} 98-137 \mathrm{~g} \\
\text { Total hip joint } \\
\text { arthroplasty }\end{array}$ & $\begin{array}{l}\text { EPO }(80,000 \mathrm{IU})+\text { oral } \\
\quad \text { iron }(n=79) \\
\text { EPO }(160,000 \mathrm{IU})+ \\
\quad \text { oral iron }(n=44) \\
\text { Placebo }+ \text { oral iron }(n= \\
\quad 78) \\
\text { Preoperative only }\end{array}$ & $\begin{array}{l}\text { Transfusion, reticulocyte count, Hb, adverse events (World Health } \\
\text { Organization definition), thromboembolic disease (proximal or } \\
\text { distal DVT, pulmonary embolism), serious adverse events }\end{array}$ \\
\hline
\end{tabular}


Table 1 continued

\begin{tabular}{|c|c|c|c|c|}
\hline Reference & Size & Population & $\begin{array}{l}\text { Intervention and } \\
\text { comparator }\end{array}$ & Outcomes \\
\hline Podesta 2000 & $n=$ & $\begin{array}{l}\text { Hct }<42 \% \\
\text { Elective cardiac surgery }\end{array}$ & $\begin{array}{l}\text { EPO }(60,000 \mathrm{IU})+\text { oral } \\
\quad \text { iron }(n=30) \\
\text { Oral iron }(n=30) \\
\text { Preoperative only }\end{array}$ & $\begin{array}{l}\text { Transfusions, Hb, hct, reticulocytes, iron values, ferritin, } \\
\text { transferrin, serologic values, side effects and adverse events } \\
\text { (hypertension, intravascular thrombosis) }\end{array}$ \\
\hline Qvist 1999 & $\begin{array}{l}n= \\
81\end{array}$ & $\begin{array}{l}\mathrm{Hb}<8.5 \mathrm{mmol} \cdot \mathrm{L}^{-1} \\
\quad\left(137 \mathrm{~g} \cdot \mathrm{L}^{-1}\right) \\
\text { Colorectal cancer } \\
\quad \text { surgery }\end{array}$ & $\begin{array}{l}\text { EPO }(\sim 70,000 \mathrm{IU})+ \\
\quad \text { oral iron }(n=38) \\
\text { Placebo }+ \text { oral iron }(n= \\
\quad 43) \\
\text { Preoperative only }\end{array}$ & $\begin{array}{l}\text { Transfusions, blood loss, hematologic parameters }(\mathrm{Hb} \text {, } \\
\text { reticulocytes, serum ferritin, transferrin, and iron), adverse } \\
\text { outcomes (i.e., deep vein thrombosis), hospital stay }\end{array}$ \\
\hline D’Ambra 1997 & $n=$ & $\begin{array}{l}\text { Hct }<45 \% \\
\text { CABG surgery }\end{array}$ & $\begin{array}{l}\text { EPO }(\sim 84,000 \mathrm{IU})+ \\
\text { oral iron }(n=63) \\
\text { EPO }(\sim 168,000 \mathrm{IU})+ \\
\quad \text { oral iron }(n=63) \\
\text { Placebo }+ \text { oral iron }(n= \\
56) \\
\text { Preoperative and } \\
\text { postoperative }\end{array}$ & $\begin{array}{l}\text { Transfusions, Hb, Hct, reticulocyte count, visual analogue energy } \\
\text { assessment, endogenous EPO levels, antibody titers, hospital } \\
\text { stay, iron stores, adverse events (reported by at least } 20 \% \text { of } \\
\text { patients), mortality }\end{array}$ \\
\hline Sowade 1997 & $n=$ & $\begin{array}{l}\text { Hct }<45 \% \\
\text { Open heart surgery }\end{array}$ & $\begin{array}{l}\text { EPO }(\sim 175,000 \mathrm{IU})+ \\
\quad \text { oral iron }(n=38) \\
\text { Placebo }+ \text { oral iron }(n= \\
\quad 38) \\
\text { Preoperative only }\end{array}$ & $\begin{array}{l}\text { Transfusions, } \mathrm{Hb}, \mathrm{Hct} \text {, iron, ferritin, transferrin saturation, adverse } \\
\text { events (no description) }\end{array}$ \\
\hline $\begin{array}{l}\text { de Andrade } \\
1996\end{array}$ & $\begin{array}{l}n= \\
290\end{array}$ & $\begin{array}{l}\mathrm{Hb}<150 \mathrm{~g} \cdot \mathrm{L}^{-1} \\
\text { Elective major } \\
\quad \text { orthopedic surgery }\end{array}$ & $\begin{array}{l}\mathrm{EPO}(\sim 105,000 \mathrm{IU})+ \\
\text { oral iron }(n=101) \\
\mathrm{EPO}(\sim 315,000 \mathrm{IU})+ \\
\text { oral iron }(n=112) \\
\text { Placebo }+ \text { oral iron }(n= \\
103) \\
\text { Preoperative and } \\
\text { postoperative }\end{array}$ & $\begin{array}{l}\text { Transfusions, blood loss, incidence and severity of adverse events } \\
\text { (i.e., pyrexia, nausea, vomiting, constipation, etc.), } \\
\text { thrombotic/vascular events, changes in ultrasonography results, } \\
\text { clinical laboratory tests, vital signs, hospital stay }\end{array}$ \\
\hline Faris 1996 & $n=$ & $\begin{array}{l}\text { No } \mathrm{Hb} \text { criteria stated } \\
\text { Major orthopedic } \\
\text { surgery }\end{array}$ & $\begin{array}{l}\text { EPO }(\sim 105,000 \mathrm{IU})+ \\
\quad \text { oral iron }(n=71) \\
\text { EPO }(\sim 315,000 \mathrm{IU})+ \\
\quad \text { oral iron }(n=60) \\
\text { Placebo }+ \text { oral iron }(n= \\
\quad 69) \\
\text { Preoperative and } \\
\text { postoperative }\end{array}$ & $\begin{array}{l}\text { Transfusions, hematologic parameters until 3-4 weeks } \\
\text { postoperatively (Hb, Hct, reticulocyte counts), all adverse } \\
\text { events (i.e., depression, chest pain, hypertension, etc.) }\end{array}$ \\
\hline Heiss 1996 & $n=$ & $\begin{array}{l}\mathrm{Hb} 90-130 \mathrm{~g} \cdot \mathrm{L}^{-1} \\
\text { Resectable colorectal } \\
\quad \text { cancer surgery }\end{array}$ & $\begin{array}{l}\text { EPO }(\sim 136,500 \mathrm{IU})+ \\
\text { oral iron }+ \text { folate }(n= \\
20) \\
\text { Placebo }+ \text { oral iron }(n= \\
10) \\
\text { Preoperative and } \\
\text { postoperative }\end{array}$ & $\begin{array}{l}\text { Transfusions, intraoperative blood loss, Hb, reticulocytes, } \\
\text { transferrin, ferritin, iron }\end{array}$ \\
\hline
\end{tabular}


Table 1 continued

\begin{tabular}{|c|c|c|c|c|}
\hline Reference & Size & Population & $\begin{array}{l}\text { Intervention and } \\
\text { comparator }\end{array}$ & Outcomes \\
\hline COPES 1993 & $\begin{array}{l}n= \\
208\end{array}$ & $\begin{array}{l}\text { Hb } 110-160 \mathrm{~g} \cdot \mathrm{L}^{-1} \\
\text { Elective hip } \\
\quad \text { replacement }\end{array}$ & $\begin{array}{l}\text { EPO }(\sim 189,000 \mathrm{IU})+ \\
\quad \text { oral iron }(n=53) \\
\text { EPO }(\sim 294,000 \mathrm{IU})+ \\
\quad \text { oral iron }(n=77) \\
\text { Oral iron }(n=78) \\
\text { Preoperative and } \\
\quad \text { postoperative }\end{array}$ & $\begin{array}{l}\text { Transfusions (intra- and postoperative), Hb, reticulocyte counts, } \\
\text { patient-reported well-being, length of hospital stay, blood } \\
\text { pressure, DVT, other clinical events }\end{array}$ \\
\hline
\end{tabular}

a The age criteria for these studies were $>12 \mathrm{yr}$. Total erythropoietin dose values were estimated for a $70 \mathrm{~kg}$ participant

$\mathrm{AKI}=$ acute kidney injury; $\mathrm{CABG}=$ coronary artery bypass graft; $\mathrm{CPB}=$ cardiopulmonary bypass; $\mathrm{DVT}=$ deep vein thrombosis; $\mathrm{EPO}=$ erythropoietin; $\mathrm{Hb}=$ hemoglobin; Hct = hematocrit; $\mathrm{ICU}=$ intensive care unit; $\mathrm{IV}=$ intravenous; $\mathrm{MI}=$ myocardial infarction; $\mathrm{PE}=$ pulmonary embolism; RBC = red blood cell

ESA, low dose ESA did not increase the risk of DVT (RR, $0.82 ; 95 \% \mathrm{CI}, 0.35$ to $1.93, \mathrm{I}^{2}=0 \%, P=0.46$, five studies). The test for subgroup differences was not different between low and high dose groups $(P=0.12)$ (ESM eFig. 12).

A sensitivity sub-analysis was performed utilizing five studies that showed a low risk of bias. From this analysis, we observed that ESA and iron therapy was more effective than iron therapy alone at reducing RBC transfusion (RR, 0.62 ; $95 \% \mathrm{CI}, 0.47$ to $0.82, \mathrm{I}^{2}=53 \%$; overall effect, $P=$ 0.001, five studies) (ESM eFig. 13). Nevertheless, given that the funnel plot showed a wedge of smaller studies missing on the side of increased transfusion, the impact of publication bias cannot be ruled out (ESM eFig. 14).

\section{Discussion}

This systematic review and meta-analysis supports the hypothesis that ESA and iron therapy is more effective than iron therapy alone at reducing perioperative $\mathrm{RBC}$ transfusion. This primary outcome was supported by additional clinically relevant secondary outcomes, which showed that ESA plus iron therapy was more effective than iron therapy alone with respect to: 1) increasing posttreatment preoperative $\mathrm{Hb}$ levels; 2) reducing the number of perioperative RBC units transfused; 3) increasing postoperative $\mathrm{Hb}$ levels; and 4) increasing reticulocyte count.

With respect to the route of iron administration, we did not detect superiority of ESA in combination with intravenous iron compared with intravenous iron alone. This may be because, compared with many studies using oral iron, there were only five studies assessing the impact of ESA and intravenous iron on RBC transfusion. It is also possible that the addition of ESAs to intravenous iron did not further reduce the incidence of RBC transfusion, as the increased efficacy of intravenous iron relative to oral iron may have negated any further therapeutic effect of ESA on optimal erythropoiesis. ${ }^{17,19}$ Data from our analysis that refutes this argument include the finding that oral or intravenous iron alone did not dramatically increase the reticulocyte count ( $1 \%$ change with treatment). This suggests that inadequate time was allowed for the maximal therapeutic effect of iron or inadequate drug dosing prevented optimal erythropoiesis in patients treated with intravenous iron alone. The increase in reticulocytosis observed in patients treated with ESA and iron $(170 \%$ change with treatment) provides biologic evidence of enhanced erythropoiesis with ESA treatment above that observed with iron alone. Further prospective trials are needed to confirm these positive findings.

Our analysis adds to the literature in this area as it reviews data from a large number of RCTS in patients undergoing cardiac and non-cardiac surgery. Previously published systematic reviews and meta-analyses ${ }^{20-22}$ were limited in that they: 1) did not include all relevant RCTs assessing the efficacy of preoperative ESA and iron therapy on RBC transfusion avoidance ${ }^{20-22}$; 2) included a large number of studies that utilized $\mathrm{PAD}^{20,21}$; 3) did not perform a formal meta-analysis ${ }^{22}$; 4) did not include data on all cardiac and non-cardiac surgical patient populations; and 5) did not include complete data on serious adverse effects associated with ESA therapy. ${ }^{20-22}$

Relative to our systematic reviews and meta-analyses, which included 25 RCTs assessing perioperative patients, previously published reviews were more limited in that they only included five (Laupacis 1998), 12 (Lin 2013) or seven RCTs ( $\mathrm{Li} 2018)^{20-22}$ that specifically addressed the comparison of ESA and iron vs iron alone as a comparator. The main reasons that we excluded trials that had previously been included in published meta-analyses are that: 1) they included studies that utilized PAD $(n=31$ 
Table 2 Characteristics of all randomized-controlled trials evaluating ESA and intravenous iron $v s$ iron studies

\begin{tabular}{|c|c|c|c|c|}
\hline Reference & Size & Population & $\begin{array}{l}\text { Intervention and } \\
\text { comparator }\end{array}$ & Outcomes \\
\hline Urena 2017 & $n=$ & $\begin{array}{l}\text { Male: } \mathrm{Hb}<130 \mathrm{~g} \cdot \mathrm{L}^{-1} \\
\text { Female: } \mathrm{Hb}<120 \mathrm{~g} \cdot \mathrm{L}^{-1} \\
\text { Transcatheter aortic valve } \\
\quad \text { implant surgery }\end{array}$ & $\begin{array}{l}\text { EPO }(35,000 \text { IU })+\text { IV iron } \\
\quad(n=48) \\
\text { Placebo }+ \text { IV iron }(n=52) \\
\text { Preoperative only }\end{array}$ & $\begin{array}{l}\text { Proportion and volume of RBC transfusion, } \mathrm{Hb} \text {, troponin, creatine kinase-MB, } \\
\text { mortality, myocardial infarction, stroke, } \mathrm{AKI} \text {, hemodialysis, atrial fibrillation }\end{array}$ \\
\hline $\begin{array}{l}\text { Bernabeu- } \\
\text { Wittel } \\
2016\end{array}$ & $n=$ & $\begin{array}{l}\mathrm{Hb} 90-120 \mathrm{~g} \cdot \mathrm{L}^{-1} \\
\text { Osteoporotic hip fracture } \\
\text { surgery }\end{array}$ & $\begin{array}{l}\text { EPO }(40,000 \mathrm{IU})+\text { IV iron } \\
\quad(n=100) \\
\text { IV iron }(n=103) \\
\text { Placebo IV iron }+ \text { EPO }(n= \\
\quad 100) \\
\text { Preoperative only }\end{array}$ & Patients transfused, survival, hemoglobinemia, health-related quality of life \\
\hline $\begin{array}{r}\text { Kateros } \\
2010\end{array}$ & $n=79$ & $\begin{array}{l}\mathrm{Hb}<130 \mathrm{~g} \cdot \mathrm{L}^{-1} \\
\text { Intertrochanteric fracture } \\
\text { repair surgery }\end{array}$ & $\begin{array}{l}\text { EPO }(\sim 200,000 \mathrm{IU})+\mathrm{IV} \\
\quad \text { iron }(n=38) \\
\text { Placebo }+ \text { IV iron }(n=41) \\
\text { Preoperative and } \\
\quad \text { postoperative }\end{array}$ & Transfusions, Hct, Hb, RBC, white blood cells, platelets \\
\hline $\begin{array}{l}\text { Kosmadakis } \\
2003\end{array}$ & $n=63$ & $\begin{array}{l}\mathrm{Hb} 85-130 \mathrm{~g} \cdot \mathrm{L}^{-1} \\
\text { Gastrointestinal tract } \\
\text { surgery to treat } \\
\text { malignancies }\end{array}$ & $\begin{array}{l}\text { EPO }(\sim 294000 \mathrm{IU})+\mathrm{IV} \\
\quad \text { iron }(n=31) \\
\text { Placebo }+ \text { IV iron }(n=32) \\
\text { Preoperative and } \\
\quad \text { postoperative }\end{array}$ & Blood loss, blood cell counts, serum chemistry, reticulocytes, ferritin, iron indices \\
\hline $\begin{array}{r}\text { Olijhoek } \\
2001^{\mathrm{a}}\end{array}$ & $n=$ & $\begin{array}{l}\mathrm{Hb} 100-130 \mathrm{~g} \cdot \mathrm{L}^{-1} \\
\text { Elective orthopedic } \\
\quad \text { surgery }\end{array}$ & $\begin{array}{l}\text { EPO }(\sim 84,000 \mathrm{IU})+\mathrm{IV} \\
\quad \text { iron }(n=29) \\
\text { EPO }(\sim 84,000 \mathrm{IU})+\text { oral } \\
\quad \text { iron }(n=29) \\
\text { Placebo + IV iron }(n=25) \\
\text { Placebo + oral iron }(n=27) \\
\text { Preoperative only }\end{array}$ & $\begin{array}{l}\mathrm{Hb}, \mathrm{RBC} \text { production, serum iron, total iron binding capacity, serum ferritin, } \\
\text { serious adverse events (including thrombotic/vascular events) }\end{array}$ \\
\hline $\begin{array}{c}\text { Kettelhack } \\
1998^{\mathrm{b}}\end{array}$ & $\begin{array}{l}n= \\
108\end{array}$ & $\begin{array}{l}\mathrm{Hb} 85-135 \mathrm{~g} \cdot \mathrm{L}^{-1} \\
\text { Right hemi-colectomy for } \\
\quad \text { carcinoma }\end{array}$ & $\begin{array}{l}\text { EPO }(\sim 200,000 \text { IU })+\text { IV } \\
\text { iron }+ \text { oral iron }(n=48) \\
\text { Placebo }+ \text { IV iron }+ \text { oral } \\
\text { iron }(n=54) \\
\text { Preoperative and } \\
\text { postoperative }\end{array}$ & Transfusions, $\mathrm{Hb}$, reticulocytes, transferrin saturation, adverse events \\
\hline Kyo $1992^{\mathrm{c}}$ & $n=95$ & $\begin{array}{l}\mathrm{Hb} 120-160 \mathrm{~g} \cdot \mathrm{L}^{-1} \\
\text { Elective cardiac surgery } \\
\quad \text { with CPB }\end{array}$ & $\begin{array}{l}\text { EPO }(3000 \mathrm{IU})+\mathrm{IV} \text { iron }+ \\
\quad \text { oral iron }(n=21) \\
\text { EPO }(6000 \mathrm{IU})+\mathrm{IV} \text { iron }+ \\
\quad \text { oral iron }(n=30) \\
\text { EPO }(9000 \mathrm{IU})+\mathrm{IV} \text { iron }+ \\
\quad \text { oral iron }(n=18) \\
\text { Oral iron }(n=26) \\
\text { Preoperative only }\end{array}$ & $\begin{array}{l}\mathrm{Hb} \text {, reticulocyte count, blood coagulation factors, liver and renal function, } \\
\text { perioperative blood loss }\end{array}$ \\
\hline
\end{tabular}

Total erythropoietin dose values were estimated for a $70 \mathrm{~kg}$ participant

${ }^{a}$ Data from this study included both an ESA + intravenous iron $v s$ intravenous iron comparison and an ESA + oral iron vs oral iron comparison. These subgroups were analyzed separately within each respective meta-analysis

${ }^{\mathrm{b}}$ Patients in this study received an ESA and oral iron therapy preoperatively, and received intravenous iron therapy on postoperative day 1

${ }^{c}$ Patients administered EPO + intravenous iron in this study were compared with patients who were administered oral iron

$\mathrm{AKI}=$ acute kidney injury; $\mathrm{CPB}=$ cardiopulmonary bypass; $\mathrm{EPO}=$ erythropoietin; $\mathrm{Hb}=$ hemoglobin; Hct = hematocrit; $\mathrm{ICU}=$ intensive care unit; $\mathrm{IV}=$ intravenous; $\mathrm{MI}=$ myocardial infarction $; \mathrm{RBC}=$ red blood cell 


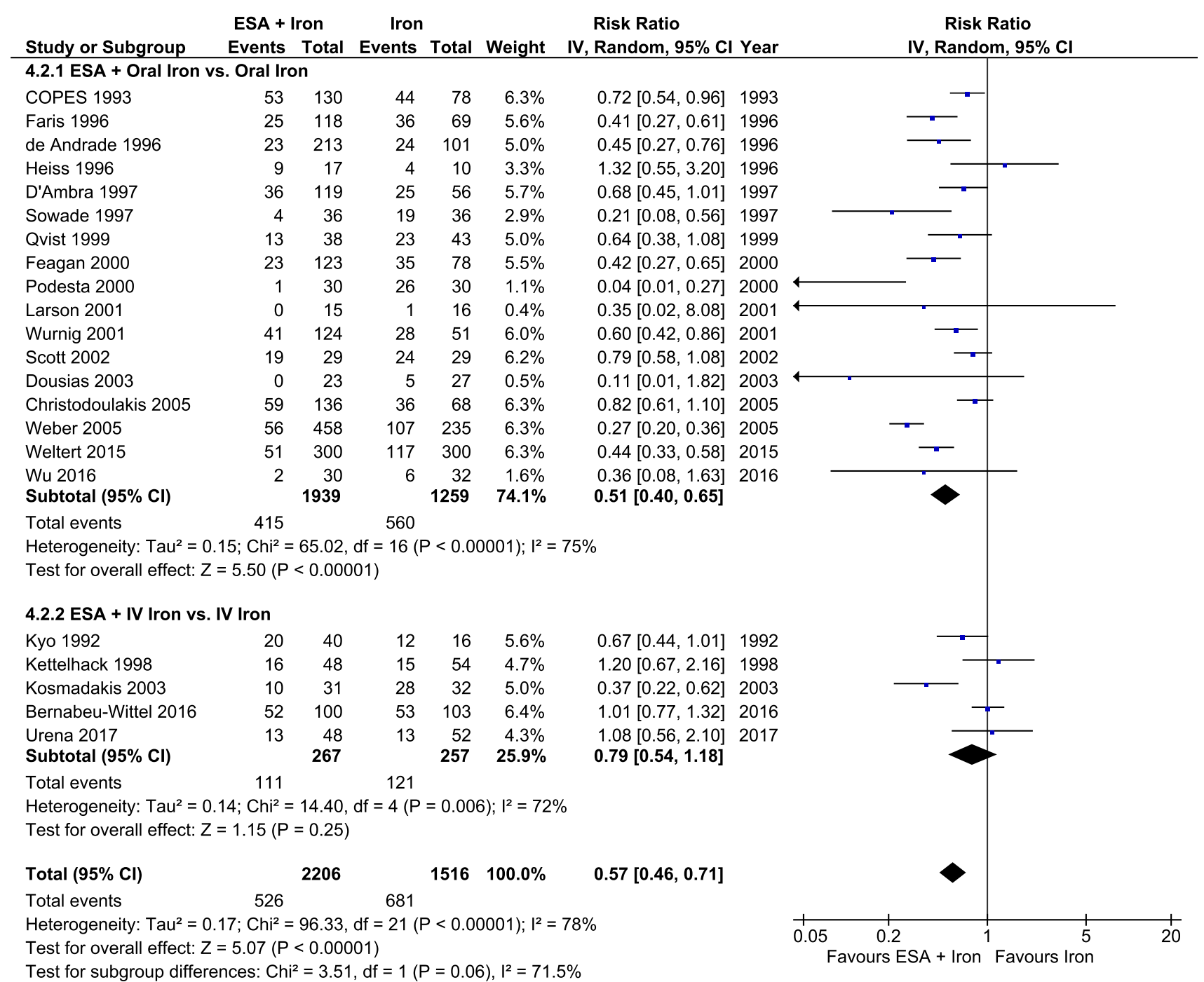

Fig. 2 Forest plot showing the effect of erythropoiesis stimulating agents (ESA) and iron $v s$ iron on number of patients transfused with red blood cells (RBCs) (primary outcome), stratified into subgroups by study interventions

trials); 2) had inappropriate comparator groups ( $n=6$ trials); or 3 ) they were not prospective RCTs ( $n=8$ trials) (ESM eTables 2 and 3). Our results are consistent with the findings of other published systematic reviews and metaanalyses assessing the efficacy of ESA therapy in surgical patients. $^{21,22}$ Nevertheless, our analysis included an additional 13 trials that were not included in the previous reviews.

With respect to assessment of adverse outcomes, our analysis did not show any evidence that ESA therapy increased the risk of postoperative mortality, stroke, MI, renal dysfunction, PE, or DVT. These findings may be clinically important as concern about the safety of ESAs in other patient populations has potentially restricted the use of ESAs in surgical patients.

Historically, early successes in utilizing ESAs to treat anemia in patients with renal failure, malignancy, or surgical patients, showed efficacy in terms of transfusion avoidance in each of these populations. ${ }^{20,58-60}$ Studies in the intensive care patient population also showed efficacy in terms of transfusion avoidance and some evidence of improved survival. ${ }^{61,62}$ Nevertheless, more recent RCTs in medical patients, which were designed to increase $\mathrm{Hb}$ levels to near $130 \mathrm{~g} \cdot \mathrm{L}^{-1}$, showed an increase in serious adverse events including thrombosis, stroke, and mortality in patients with medical comorbidities. ${ }^{24-26}$ Furthermore, studies in perioperative patients showed a potential increased risk of thrombosis. ${ }^{35,49,50}$ The outcome data from these and other trials contributed to an ESA black box warning for increased risk of death and other serious adverse events for patients with renal failure or cancer with ESA therapy, and called for a restriction of the maximal target $\mathrm{Hb}(<$ $120 \mathrm{~g} \cdot \mathrm{L}^{-1}$ ) to be achieved with ESA therapy.

Regarding the designation of high and low dose ESA levels above or below 80,000 IU, suggested dosing 


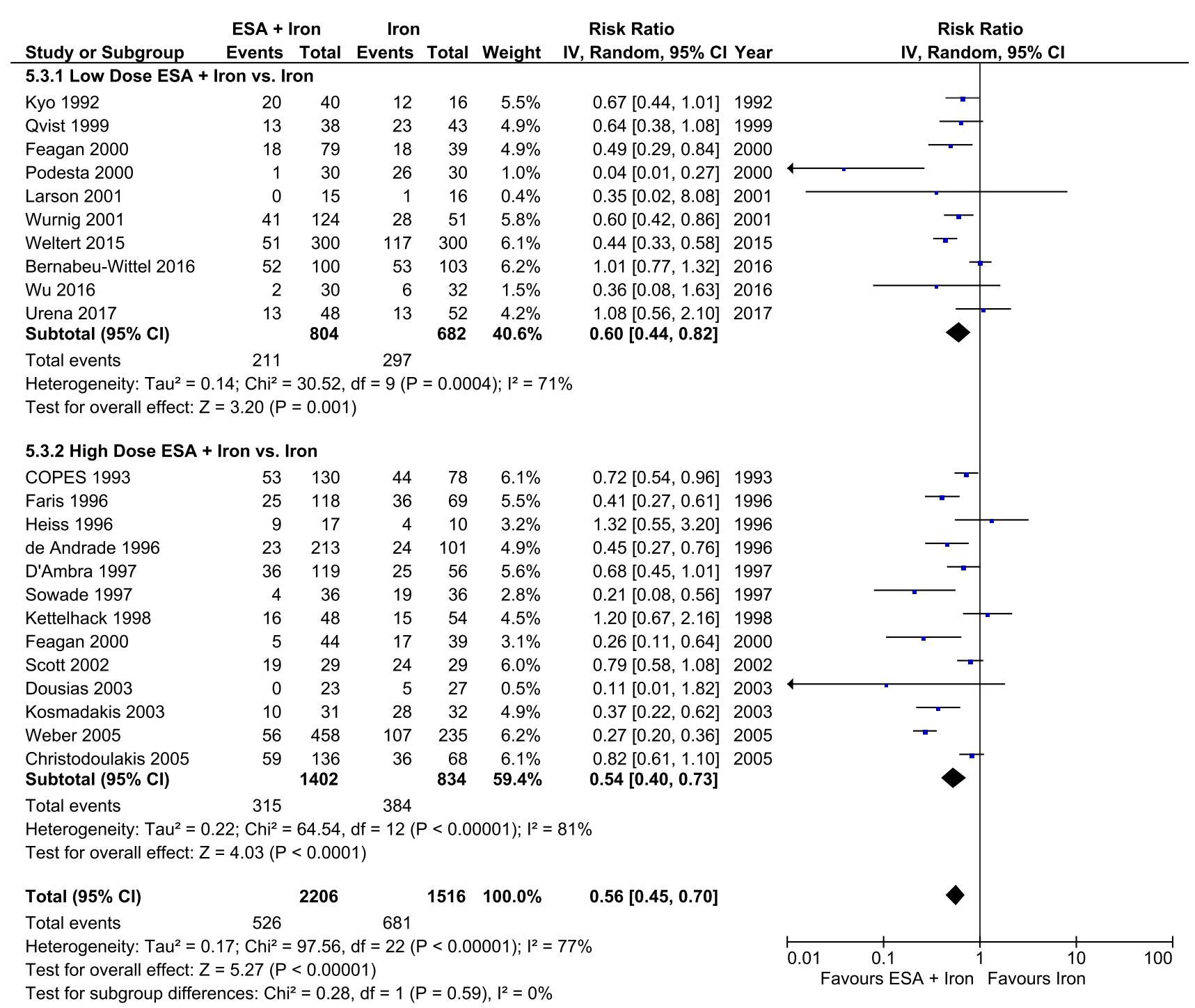

Fig. 3 Forest plot showing the effect of erythropoiesis stimulating agents (ESA) and iron $v s$ iron on number of patients transfused with red blood cells (RBCs) (primary outcome), stratified by low dose $(\leq 80,000 \mathrm{IU}) v s$ high dose ESA $(>80,000 \mathrm{IU})$

regiments for ESA in the perioperative period include up to three weekly doses of $600 \mathrm{IU} \cdot \mathrm{kg}^{-1}$ subcutaneously (maximum total of $126,000 \mathrm{IU} / 70 \mathrm{~kg}$ patient) or 300 $\mathrm{IU} \cdot \mathrm{kg}^{-1}$ subcutaneously given daily for ten consecutive days (210,000 IU per $70 \mathrm{~kg}$ patient). Given that a common pragmatic dosing of ESA includes up to two weekly doses of 40,000 IU preoperatively, we arbitrarily assigned a value of $<80,000$ IU as the "low dose" regime and $\geq 80,000 \mathrm{IU}$ as the "high dose" regime. In the case of treatment of anemia associated with malignancy, the individual dose of ESA used may be much lower than $80,000 \mathrm{IU}$, in order to avoid potential thrombotic complications.

Our data did not show a clear increased risk of DVT when all studies were combined. Nevertheless, when studies were stratified by ESA dose, high dose ESA ( $>$ $80,000 \mathrm{IU})$ showed a trend toward an increase in risk of
DVT. The observed increase in DVT risk was largely attributed to results from three large RCTs. ${ }^{35,49,50}$ Stowell et al. studied 680 spine surgery patients. ${ }^{35}$ They observed that the incidence of DVT was $4.7 \%$ in patients that received high dose EPO (600 IU $\cdot \mathrm{kg}^{-1} \cdot \mathrm{day}^{-1}$ for 12 days $\sim 504,000 \mathrm{IU} / 70 \mathrm{~kg}$ patient) $v s 2.1 \%$ in the standard care group (between group difference 2.6, 97.5\% upper confidence limit 5.4\%). Nevertheless, this study did not utilize standard DVT prophylaxis; therefore, it is difficult to interpret these results. The COPES trial compared two doses of EPO with a placebo group, where both EPO groups were administered relatively high doses of EPO (300 IU $\cdot \mathrm{kg}^{-1}$ per day for nine days $\sim 189,000 \mathrm{IU} / 70 \mathrm{~kg}$ patient, or $300 \mathrm{IU} \cdot \mathrm{kg}^{-1}$ for 14 days $\sim 294,000 \mathrm{IU} / 70 \mathrm{~kg}$ patient). ${ }^{63}$ Although, this trial did not reveal a significant difference between any of the three groups, pooling both 


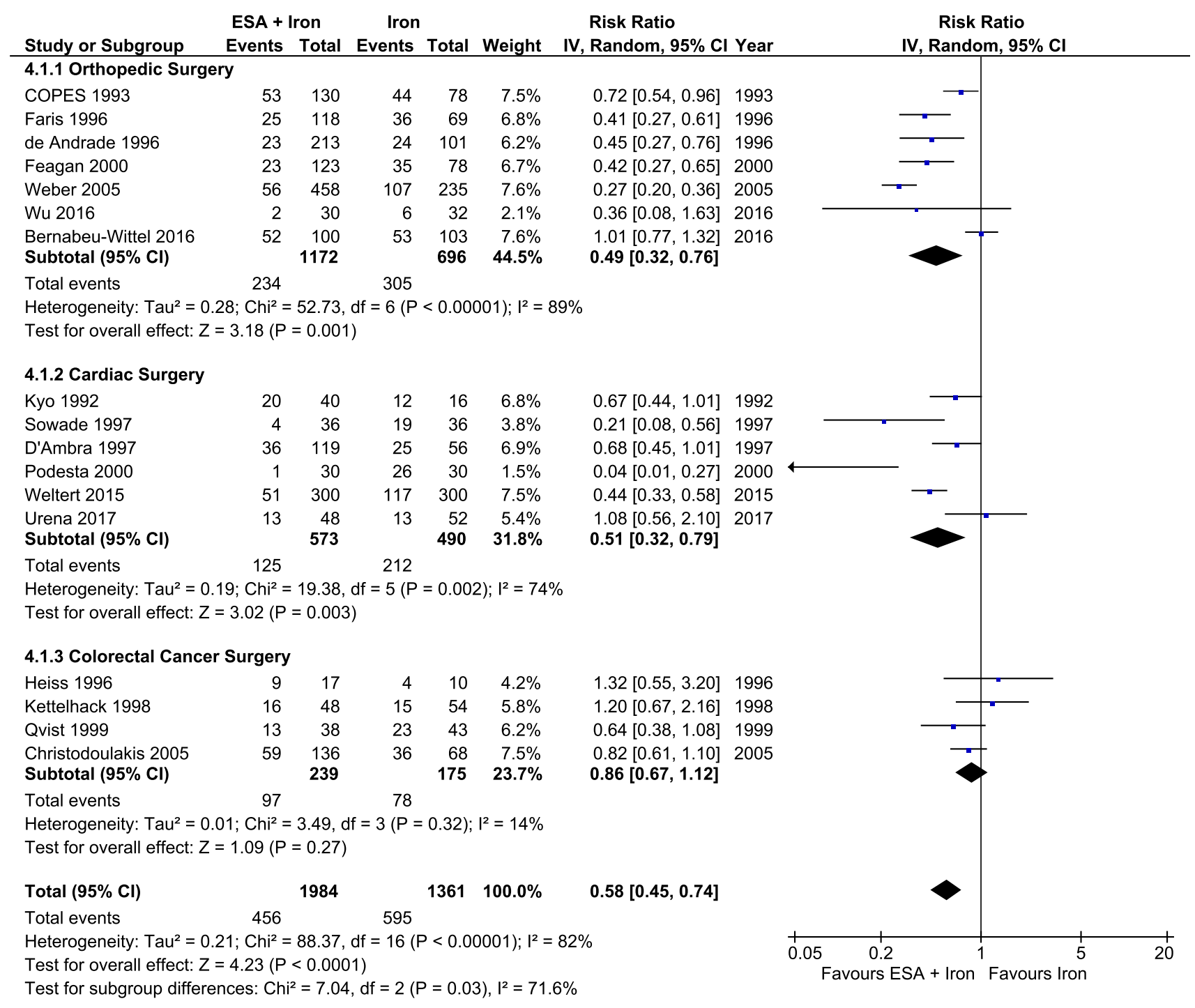

Fig. 4 Forest plot showing the effect of erythropoiesis stimulating agents (ESA) and iron $v s$ iron on number of patients transfused with red blood cells (RBCs) (primary outcome), stratified by type of surgery

EPO groups showed a trend to an increased risk of DVT. Similarly, an RCT in orthopedic surgery using high doses of EPO (100 IU $\cdot \mathrm{kg}^{-1}$ or $300 \mathrm{IU} \cdot \mathrm{kg}^{-1} \cdot \mathrm{day}^{-1}$ for 15 days) suggested a possible risk for DVT in the group receiving the highest does of EPO (10\% of $300 \mathrm{IU} \cdot \mathrm{kg}^{-1} \mathrm{EPO} ; 3 \%$ of $100 \mathrm{IU} \cdot \mathrm{kg}^{-1} \mathrm{EPO} ; 5 \%$ of placebo patients $) .{ }^{49}$ In addition, a large RCT in critically ill patients showed an increased risk of thrombosis with EPO therapy (HR, 1.41; 95\% CI, 1.06 to $1.86, P=0.008) .{ }^{62}$ Thus, there is evidence that high dose ESA may increase the number of clinically important thrombotic events. Notably, we did not observe any evidence of increase in DVT incidence when studies utilized low dose ESA (i.e., < 80,000 IU): a commonly utilized dose in blood conservation programs with evidence of efficacy in terms of avoiding RBC transfusion.
Subgroup analysis in different surgical groups showed that patients undergoing cardiac or orthopedic surgical procedures showed a transfusion-sparing effect when ESA was added to iron therapy. This effect was not observed in patients undergoing colorectal surgery. These differences may reflect a higher incidence of ACI in patients undergoing cardiac and orthopedic surgery where ESA contributes to releasing sequestered iron stores. By contrast, patients with colorectal disease may experience a higher degree of iron deficiency in which replenishing iron may be as effective as ESA + iron in this group.

Strengths of this review include a focused and comprehensive literature search and a consideration of a broad range of surgical populations and important clinical outcomes. As a result, we were able to identify a large 
number of RCTs that compared ESA + iron $v s$ iron alone. Formal analysis of these studies showed that addition of ESAs can be safely used to reduce RBC transfusion at doses compatible with that used in perioperative medicine. Nevertheless, there are limitations to our review. Our broad inclusion criteria likely contributed to the high degree of statistical heterogeneity amongst studies reporting RBC transfusion rates. This may be because studies were included that involved treatment of patients that were either anemic or non-anemic at baseline. Other contributing factors include varying types of surgery, different standards of clinical care, widely varying doses of ESA, variability in the treatment period, and lack of consistent predefined transfusion triggers. In general, studies utilizing oral iron with ESA were performed at an earlier time than those with intravenous iron, introducing the possibility that changes in practice over time may have influenced the outcomes. To address the observed heterogeneity, sensitivity analyses were performed by including studies with a low risk of bias. This analysis reduced clinical heterogeneity and maintained the significance of the impact of ESA on RBC transfusion avoidance.

By contrast, low heterogeneity was observed for all safety outcomes including mortality, stroke, MI, renal dysfunction, and $\mathrm{PE}$ - suggesting no increased risk in adverse events with ESA therapy. We did identify a potential increased risk of DVT with high dose ESA; nevertheless, this is not necessarily relevant to our patient population as these doses of ESA are not currently used in elective surgical patients enrolled in most blood management programs.

While the primary outcome of RBC transfusion was shown to be impacted by treatment with EPO and iron relative to iron alone, the robustness of this finding is tempered by the high degree of study heterogeneity and the possibility of publication bias. This study is inadequately powered to clearly define the impact of EPO and iron therapy on adverse outcomes including death, stroke, MI, AKI, and thrombosis. Future progress will require the design and completion of a large multicentre RCT to determine the impact of optimal anemia treatment on event-free patient survival.

In conclusion, the results of this meta-analysis suggest that ESA and iron therapy combined may be more effective than iron therapy alone in reducing perioperative RBC utilization and increasing postoperative $\mathrm{Hb}$ levels. Furthermore, ESA and iron therapy did not increase the risk of mortality, stroke, MI, renal dysfunction PE, or DVT compared with iron therapy alone. Large RCTs are needed to further test the hypothesis that ESA and iron therapy can safely reduce RBC transfusion and improve patient recovery without the risk of serious adverse outcomes.
Conflicts of interest None declared.

Editorial responsibility This submission was handled by Dr. Philip M. Jones, Associate Editor, Canadian Journal of Anesthesia.

Author contributions Tiffanie Kei, Nikhil Mistry, Gerard Curley, Katerina Pavenski, Nadine Shehata, Kevin Thorpe, Tom A. Schweizer, Sarah Ward, C. David Mazer, and Gregory M.T. Hare contributed substantially to the conception and design of the study. Tiffanie Kei, Nikhil Mistry, Gerard Curley, C. David Mazer, and Gregory M.T. Hare contributed substantially to the acquisition of data. Tiffanie Kei, Nikhil Mistry, Gerard Curley, Katerina Pavenski, Nadine Shehata, Rosa Maria Tanzini, Marie-France Gauthier, Kevin Thorpe, Tom A. Schweizer, Sarah Ward, C. David Mazer, and Gregory M.T. Hare contributed to the analysis of data. Tiffanie Kei, Nikhil Mistry, Gerard Curley, Katerina Pavenski, Nadine Shehata, Rosa Maria Tanzini, Marie-France Gauthier, Kevin Thorpe, Tom A. Schweizer, Sarah Ward, C. David Mazer, and Gregory M.T. Hare contributed substantially to the interpretation of data. All authors contributed to drafting, revising, and adding important intellectual content to the manuscript.

Funding support Dr. Hare and Dr. Mazer are supported by University of Toronto, Department of Anesthesia Merit Awards. Dr. Hare received support from the Academic Health Science Centre Alternative Funding Plan (SMH-15-012). Dr. Curley has received Innovation Fund support from Royal College of Surgeons in Ireland. Dr. Shehata is supported by a Canadian Institute of Health Research/ Canadian Blood Services New Investigator Award. The authors would like to acknowledge Mr. David Lightfoot for his assistance with the formal literature search.

\section{References}

1. Karkouti K, Wijeysundera DN, Beattie WS, Reducing Bleeding in Cardiac Surgery (RBC) Investigators. Risk associated with preoperative anemia in cardiac surgery: a multicenter cohort study. Circulation 2008; 117: 478-84.

2. Kulier A, Levin J, Moser R, et al. Impact of preoperative anemia on outcome in patients undergoing coronary artery bypass graft surgery. Circulation 2007; 116: 471-9.

3. Beattie WS, Karkouti K, Wijeysundera DN, Tait G. Risk associated with preoperative anemia in noncardiac surgery: a single-center cohort study. Anesthesiology 2009; 110: 574-81.

4. Carson JL, Poses RM, Spence RK, Bonavita G. Severity of anaemia and operative mortality and morbidity. Lancet 1988; 1 : 727-9.

5. Carson JL, Duff A, Poses RM, et al. Effect of anaemia and cardiovascular disease on surgical mortality and morbidity. Lancet 1996; 348: 1055-60.

6. Wu WC, Schifftner TL, Henderson WG, et al. Preoperative hematocrit levels and postoperative outcomes in older patients undergoing noncardiac surgery. JAMA 2007; 297: 2481-8.

7. Leichtle SW, Mouawad NJ, Lampman R, Singal B, Cleary RK. Does preoperative anemia adversely affect colon and rectal surgery outcomes? J Am Coll Surg 2011; 212: 187-94.

8. Musallam KM, Tamim HM, Richards T, et al. Preoperative anaemia and postoperative outcomes in non-cardiac surgery: a retrospective cohort study. Lancet 2011; 378: 1396-407.

9. Klein AA, Collier TJ, Brar MS, et al. The incidence and importance of anaemia in patients undergoing cardiac surgery in 
the UK - the first Association of Cardiothoracic Anaesthetists national audit. Anaesthesia 2016; 71: 627-35.

10. Feng S, Machina M, Beattie WS. Influence of anaemia and red blood cell transfusion on mortality in high cardiac risk patients undergoing major non-cardiac surgery: a retrospective cohort study. Br J Anaesth 2017; 118: 843-51.

11. Munoz M, Laso-Morales MJ, Gomez-Ramirez S, Cadellas M, Nunez-Matas MJ, Garcia-Erce JA. Pre-operative haemoglobin levels and iron status in a large multicentre cohort of patients undergoing major elective surgery. Anaesthesia 2017; 72: 826-34.

12. Shander A, Goodnough LT, Javidroozi M, et al. Iron deficiency anemia-bridging the knowledge and practice gap. Transfus Med Rev 2014; 28: 156-66.

13. Goodnough $L T$, Shander A. Update on erythropoiesis-stimulating agents. Best Pract Res Clin Anaesthesiol 2013; 27: 121-9.

14. Goodnough LT, Shander A. Patient blood management. Anesthesiology 2012; 116: 1367-76.

15. Elhenawy AM, Meyer SR, Bagshaw SM, MacArthur RG, Carroll $L J$. Role of preoperative intravenous iron therapy to correct anemia before major surgery: study protocol for systematic review and meta-analysis. Syst Rev 2015; 4: 29.

16. Hallet $J$, Hanif A, Callum $J$, et al. The impact of perioperative iron on the use of red blood cell transfusions in gastrointestinal surgery: a systematic review and meta-analysis. Transfus Med Rev 2014; 28: 205-11.

17. Perelman I, Winter $R$, Sikora L, Martel $G$, Saidenberg E, Fergusson D. The efficacy of postoperative iron therapy in improving clinical and patient-centered outcomes following surgery: a systematic review and meta-analysis. Transfus Med Rev 2018; 32: 89-101.

18. Yang $Y, L i H, L i B$, Wang $Y$, Jiang $S$, Jiang $L$. Efficacy and safety of iron supplementation for the elderly patients undergoing hip or knee surgery: a meta-analysis of randomized controlled trials. J Surg Res 2011; 171: e201-7.

19. Biboulet $P$, Bringuier $S$, Smilevitch $P$, et al. Preoperative epoetinalpha with intravenous or oral iron for major orthopedic surgery: a randomized controlled trial. Anesthesiology 2018; 129: 710-20.

20. Laupacis A, Fergusson D. Erythropoietin to minimize perioperative blood transfusion: a systematic review of randomized trials. The International Study of Peri-operative Transfusion (ISPOT) Investigators. Transfus Med 1998; 8: 309-17.

21. Li Y, Yin $P, L v H$, Meng $Y$, Zhang $L$, Tang $P$. A meta-analysis and systematic review evaluating the use of erythropoietin in total hip and knee arthroplasty. Ther Clin Risk Manag 2018; 14: 1191-204.

22. Lin DM, Lin ES, Tran MH. Efficacy and safety of erythropoietin and intravenous iron in perioperative blood management: a systematic review. Transfus Med Rev 2013; 27: 221-34.

23. Richards $T$, Clevenger B, Keidan $J$, et al. PREVENTT: preoperative intravenous iron to treat anaemia in major surgery: study protocol for a randomised controlled trial. Trials 2015; 16 : 254.

24. Drueke TB, Locatelli $F$, Clyne $N$, et al. Normalization of hemoglobin level in patients with chronic kidney disease and anemia. N Engl J Med 2006; 355: 2071-84.

25. Pfeffer MA, Burdmann EA, Chen CY, et al. A trial of darbepoetin alfa in type 2 diabetes and chronic kidney disease. N Engl J Med 2009; 361: 2019-32.

26. Singh AK, Szczech L, Tang KL, et al. Correction of anemia with epoetin alfa in chronic kidney disease. N Engl J Med 2006; 355 : 2085-98.

27. Higgins JP, Green S. Cochrane Collaboration. Cochrane Handbook for Systematic Reviews of Interventions. NJ: WileyBlackwell; 2008 .

28. Higgins JP, Altman DG. Assessing risk of bias in included studies. In: Higgins JP, Green S (Eds). Cochrane Handbook for
Systematic Reviews of Interventions: John Wiley \& Sons, Ltd; 2008: 187-241.

29. DerSimonian R, Laird N. Meta-analysis in clinical trials. Control Clin Trials 1986; 7: 177-88.

30. Higgins JP, Thompson SG, Deeks JJ, Altman DG. Measuring inconsistency in meta-analyses. BMJ 2003; 327: 557-60.

31. Hozo SP, Djulbegovic B, Hozo I. Estimating the mean and variance from the median, range, and the size of a sample. BMC Med Res Methodol 2005; 5: 13.

32. Richardson $M$, Garner P, Donegan S. Interpretation of subgroup analyses in systematic reviews: a tutorial. Clin Epidemiol Global Health 2018; DOI: https://doi.org/10.1016/j.cegh.2018. 05.005 .

33. Wu YG, Zeng $Y$, Shen B, et al. Combination of erythropoietin and tranexamic acid in bilateral simultaneous total hip arthroplasty: a randomised, controlled trial. Hip Int 2016; 26: 331-7.

34. Weltert $L$, Rondinelli $B$, Bello $R$, et al. A single dose of erythropoietin reduces perioperative transfusions in cardiac surgery: results of a prospective single-blind randomized controlled trial. Transfusion 2015; 55: 1644-54.

35. Stowell CP, Jones SC, Enny C, Langholff W, Leitz G. An openlabel, randomized, parallel-group study of perioperative epoetin alfa versus standard of care for blood conservation in major elective spinal surgery: safety analysis. Spine (Phila Pa 1976) 2009; 34: 2479-85.

36. Weber EW, Slappendel R, Hemon $Y$, et al. Effects of epoetin alfa on blood transfusions and postoperative recovery in orthopaedic surgery: the European Epoetin Alfa Surgery Trial (EEST). Eur J Anaesthesiol 2005; 22: 249-57.

37. Christodoulakis M, Tsiftsis DD, Hellenic Surgical Oncology Perioperative EPO Study Group. Preoperative epoetin alfa in colorectal surgery: a randomized, controlled study. Ann Surg Oncol 2005; 12: 718-25.

38. Dousias V, Paraskevaidis E, Dalkalitsis $N$, Tsanadis $G$, Navrozoglou I, Lolis D. Recombinant human erythropoietin in mildly anemic women before total hysterectomy. Clin Exp Obstet Gynecol 2003; 30: 235-8.

39. Scott SN, Boeve TJ, McCulloch TM, Fitzpatrick KA, Karnell LH. The effects of epoetin alfa on transfusion requirements in head and neck cancer patients: a prospective, randomized, placebocontrolled study. Laryngoscope 2002; 112: 1221-9.

40. Wurnig C, Schatz K, Noske $H$, et al. Subcutaneous low-dose epoetin beta for the avoidance of transfusion in patients scheduled for elective surgery not eligible for autologous blood donation. Eur Surg Res 2001; 33: 303-10.

41. Larson B, Bremme $K$, Clyne N, Nordstrom L. Preoperative treatment of anemic women with epoetin beta. Acta Obstet Gynecol Scand 2001; 80: 559-62.

42. Qvist $N$, Boesby $S$, Wolff B, Hansen $C P$. Perioperative administration of recombinant human erythropoietin in colorectal cancer surgery. A prospective, randomized, doubleblind placebo controlled study (Danish). Ugeskr Laeger 2000; 162: $355-8$.

43. Podesta A, Carmagnini E, Parodi E, et al. Elective coronary and valve surgery without blood transfusion in patients treated with recombinant human erythropoietin (epoetin-alpha). Minerva Cardioangiol 2000; 48: 341-7.

44. Feagan BG, Wong CJ, Kirkley A, et al. Erythropoietin with iron supplementation to prevent allogeneic blood transfusion in total hip joint arthroplasty. A randomized, controlled trial. Ann Intern Med 2000; 133: 845-54.

45. Sowade $O$, Warnke $H$, Scigalla $P$, et al. Avoidance of allogeneic blood transfusions by treatment with epoetin beta (recombinant human erythropoietin) in patients undergoing open-heart surgery. Blood 1997; 89: 411-8. 
46. D'Ambra MN, Gray RJ, Hillman R, et al. Effect of recombinant human erythropoietin on transfusion risk in coronary bypass patients. Ann Thorac Surg 1997; 64: 1686-93.

47. Heiss MM, Tarabichi A, Delanoff $C$, et al. Perisurgical erythropoietin application in anemic patients with colorectal cancer: a double-blind randomized study. Surgery 1996; 119: 523-7.

48. Faris PM, Ritter MA, Abels RI. The effects of recombinant human erythropoietin on perioperative transfusion requirements in patients having a major orthopaedic operation. The American Erythropoietin Study Group. J Bone Joint Surg Am 1996; 78: 6272.

49. de Andrade JR, Jove M, Landon G, Frei D, Guilfoyle M, Young $D C$. Baseline hemoglobin as a predictor of risk of transfusion and response to epoetin alfa in orthopedic surgery patients. Am J Orthop (Belle Mead NJ) 1996; 25: 533-42.

50. Effectiveness of perioperative recombinant human erythropoietin in elective hip replacement. Canadian Orthopedic Perioperative Erythropoietin Study Group. Lancet 1993; 341: 1227-32.

51. Urena $M$, Del Trigo $M$, Altisent OA, et al. Combined erythropoietin and iron therapy for anaemic patients undergoing transcatheter aortic valve implantation: the EPICURE randomised clinical trial. EuroIntervention 2017; 13: 44-52.

52. Bernabeu-Wittel M, Romero M, Ollero-Baturone $M$, et al. Ferric carboxymaltose with or without erythropoietin in anemic patients with hip fracture: a randomized clinical trial. Transfusion 2016; 56: 2199-211.

53. Kateros K, Sakellariou VI, Sofianos IP, Papagelopoulos PJ. Epoetin alfa reduces blood transfusion requirements in patients with intertrochanteric fracture. J Crit Care 2010; 25: 348-53.

54. Kosmadakis N, Messaris E, Maris A, et al. Perioperative erythropoietin administration in patients with gastrointestinal tract cancer: prospective randomized double-blind study. Ann Surg 2003; 237: 417-21.

55. Olijhoek $G$, Megens $J G$, Musto $P$, et al. Role of oral versus IV iron supplementation in the erythropoietic response to rHuEPO: a randomized, placebo-controlled trial. Transfusion 2001; 41: 95763.

56. Kettelhack C, Hones $C$, Messinger D, Schlag PM. Randomized multicentre trial of the influence of recombinant human erythropoietin on intraoperative and postoperative transfusion need in anaemic patients undergoing right hemicolectomy for carcinoma. Br J Surg 1998; 85: 63-7.

57. Kyo S, Omoto R, Hirashima K, Eguchi S, Fujita T. Effect of human recombinant erythropoietin on reduction of homologous blood transfusion in open-heart surgery. A Japanese multicenter study. Circulation 1992; 86: II413-8.

58. Bohlius J, Wilson J, Seidenfeld J, et al. Recombinant human erythropoietins and cancer patients: updated meta-analysis of 57 studies including 9353 patients. J Natl Cancer Inst 2006; 98: 70814.

59. Bohlius J, Wilson J, Seidenfeld J, et al. Erythropoietin or darbepoetin for patients with cancer. Cochrane Database Syst Rev 2006; 3: CD003407.

60. Eschbach JW, Abdulhadi MH, Browne JK, et al. Recombinant human erythropoietin in anemic patients with end-stage renal disease. Results of a phase III multicenter clinical trial. Ann Intern Med 1989; 111: 992-1000.

61. Corwin HL, Gettinger A, Pearl RG, et al. Efficacy of recombinant human erythropoietin in critically ill patients: a randomized controlled trial. JAMA 2002; 288: 2827-35.

62. Corwin HL, Gettinger A, Fabian TC, et al. Efficacy and safety of epoetin alfa in critically ill patients. N Engl J Med 2007; 357: 965-76.

63. Laupacis A, Feagan B, Wong C. Effectiveness of perioperative recombinant human erythropoietin in elective hip replacement. Canadian Orthopedic Perioperative Erythropoietin Study Group. Lancet 1993; 342: 378.

Publisher's Note Springer Nature remains neutral with regard to jurisdictional claims in published maps and institutional affiliations. 